Original article

\title{
"Prevalence and distribution of bone defects associated with moderate and severe periodontitis patients"
}

\author{
Shivani Sachdeva ${ }^{\mathrm{a}, *}$, M.B. Phadnaik ${ }^{\mathrm{b}}$, Amit Mani $^{\mathrm{a}}$, Harish Saluja ${ }^{\mathrm{a}}$, Mukund Singh ${ }^{\mathrm{c}}$ \\ ${ }^{a}$ Pravara Institute of Medical Sciences, Rural Dental College and Hospital, India \\ ${ }^{\mathrm{b}}$ Governmentl Dental College Nagpur, India \\ ${ }^{\mathrm{c}}$ I.T.S. Ghaziabad, India
}

\section{A R T I C L E I N F O}

The following research article has been presented at 2nd International congress of World Academy of Growth Factors and Stem cells in Dentistry (WAGRO).

Keywords:

Bone defects

Periodontitis

Prevalence

Interradicular defects

Infrabony and ramp

\begin{abstract}
A B S T R A C T
Aim: As periodontal disease is major public dental health problem and knowledge of the osseous defects is useful to arrive at diagnosis, to assess prognosis and to plan the treatment. The purpose of this study was to analyze the prevalence and distribution of different forms of bone defects in Indian population.

Material and methods: The study population comprised of forty four patients with moderate to advanced chronic periodontitis. A total sample of 1041 teeth were explored surgically and classified as the primary level of classification system made for the study.

Results: Among 1010 defects the prevalence of bone defects was 97\%. Mandibular posteriors had highest occurrence of craters, they accounted for half of the total craters in the present study population. Majority of other bone defects were found in posterior segments than the anterior segments. Grade IV furcation involvement was not present for the present study sample.

Conclusion: The present study supports the contention that the form of alveolar bone defects reflects the original morphology of the alveolar bone.
\end{abstract}

\section{Introduction}

"Until you can count it, weigh it, or express it in a quantitative fashion, you have scarcely begun to think about the problem in a scientific fashion." Periodontal disease is one of the most widespread diseases of mankind. No nation and no area of the world are free from it, affecting almost the entire adult population (Burt 1994). ${ }^{1}$

Alveolar bone destruction is primarily caused by bacterial plaque that leads to inflammation of periodontal tissue. Bacterial plaque induces an increase of osteoclast formation and activity through direct or indirect mechanisms. Equilibrium between bone formation and bone resorption is thus shifted to favor the latter and results in the destruction of alveolar bone. ${ }^{2}$ The pathway of extension is crucial because it affects the form of the bone defect. The goal of applying the epidemiological principles to evaluate the prevalence and distribution of these alveolar bone defects, surgically explored under direct illumination for patients having moderate or severe chronic periodontitis within the age range of $30-50$ years.

Also, an attempt has been made to describe the first level of classification for periodontal bone defects. Accordingly, defects were classified into suprabony defects, infrabony defects, other bone defects and interradicular defects among arches and segments of oral cavity. The present study is descriptive cross-sectional epidemiological study.

\section{Material And Methods}

The prevalence and distribution of bone defects in forty-four patients within the age range of 30-50 years comprising of 21 males and 23 females having moderate to severe chronic periodontitis was selected. The study population provided the sample of 1041 teeth which were surgically explored and the defects were designated according to the classification criteria followed. In the exclusion criteria patients with any systemic disease, aggressive periodontitis, smokers and with probing pocket depth of $\leq 4 \mathrm{~mm}$ after non surgical periodontal therapy were excluded.

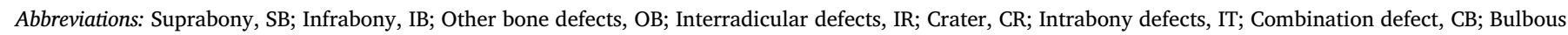
bony contour, BB; Ledge, LD; Reversed architecture, RA; Fenestration, FN; Dehiscence, DH; Trench, TR; Ramp, RM

* Corresponding author. Department of Periodontics, Pravara Rural Dental College and Hospital, India.

E-mail address: dr.shivani19@gmail.com (S. Sachdeva). 


\subsection{Method of collecting data}

A given sample of 1041 teeth was explored from the patients referred to out patient department of periodontics in government dental college, Aurangabad. The patients were examined and diagnosis of moderate and severe chronic periodontitis was made after detailed clinical and standardized radiological examination. Informed consent was obtained from all patient participants and protocol was approved by Institutional ethical committee \& Maharashtra University of Health Sciences, Nashik, Maharashtra (India).

\subsection{Procedure}

The non-surgical treatment consisting of plaque control instructions, scaling and root planing under local anesthesia was carried out. Reevaluation of the initial therapy was performed 6-8 weeks later and then patients were considered for periodontal surgical treatment. Periodontal surgery was performed and the osseous defects were explored and recorded both in maxilla and mandible under good illumination.

\subsection{Classification and description of periodontal bone defects}

In deciding, the morphology of the periodontal bone defects the following classification system has been followed:

\subsubsection{Suprabony defects}

2.3.1.1. Infrabony defects

a. Craters

b. Intrabony defects (1-, 2-, 3-, 4- wall defect and combination)

\subsubsection{Other bone defects}

a. Bulbous bone contour

b. Ledge

c. Reversed architecture

d. Fenestration

e. Dehiscence

f. Trench

g. Ramp (facial/lingual or palatal)

\subsubsection{Interradicular defects according to Glickman; $1953^{2}$}
a. Grade I
b. Grade II
c. Grade III
d. Grade IV

\subsection{Collection, comparison and statistical analysis}

Simple random sampling was carried out. All the raw data was assembled in master chart by counting the suprabony (SB), infrabony (IB), other bone defects (OB) and interradicular defects (IR) for the study population. The data from the master chart was tabulated and segregated as per the arches (maxilla and mandible) and segments (maxillary anterior, maxillary posterior, mandibular anterior and mandibular posterior).

\subsubsection{Part A: study population}

It constituted evaluation of prevalence and distribution of $\mathrm{SB}, \mathrm{IB}, \mathrm{OB}$ and IR defects within study population. In the present study the SB, IB and $\mathrm{OB}$ defects were considered as absolute defects; while, the IR defects were considered as relative defects. Absolute intergroup comparison for the prevalence of all the defects was done by percentages for the study population.

\subsubsection{Part B: arch}

It constituted evaluation of prevalence and distribution of $\mathrm{SB}, \mathrm{IB}, \mathrm{OB}$ and IR defects among maxilla and mandible. The prevalence of IR defects among multirooted teeth was also calculated for 2 different arches because it facilitated us in making comparison for the possibility of occurrences in IR defects among multirooted teeth (Lorato 1970). ${ }^{3}$

\subsubsection{Part C: segments}

It constituted evaluation of the distribution of SB, IB and OB defects between anterior (maxillary and mandibular) and posterior (maxillary and mandibular) was assessed and compared for different segments.

Statistical software 'Graph Pad Prism version 5' was used for the analysis of data. The percentage and distribution of different defects were evaluated for statistical significance using Chi-square test and Fisher exact test.

\section{Results}

In the present study 1010 defects were found. The prevalence of bone defects was $97 \%$. These defects were associated with 979 teeth. Hence, the prevalence of teeth with absolute defects was $94 \%$. These 1010 defects were the absolute defects which comprised of SB, IB and OB defects. Out of these 1010 defects, 184 defects were associated with single tooth, while 826 defects were associated with more than single tooth.

In the present study, interradicular defects were calculated separately. Total 404 multirooted teeth were explored and out of that, 254 IR defects were observed comprising of Grade I, II and III furcation involvement. Grade IV furcation involvement was not observed among the relative IR defects. The prevalence of IR defects among 404 multirooted teeth examined was $62.8 \%$. The prevalence for IR defects in maxilla was $57.5 \%$, while in mandible it was $72.7 \%$.

\subsection{Part A: study population}

Among 1010 defects, SB defects comprised 643 defects (63.7\%), IB defects comprised 314 defects (31.1\%) and OB defects comprised 53 defects (5.2\%). CR comprised of 147 defects (46.8\%) and IT defects comprised of 167 defects (53.2\%).

The prevalence and distribution of IT defects i.e. 1-, 2-, 3-, 4- wall and CB defects was as follows: 33 defects (19.7\%), 68 defects $(40.7 \%)$, 36 defects (21.6\%), 23 defects $(13.8 \%)$ and 7 defects $(4.2 \%)$, respectively.

53 OB defects i.e. BB, LD, RA, FN, DH, TR and RM was as follows 14 (26.4\%), 22 (41.5\%), 3 (5.7\%), 0, 4 (7.6\%), $6(11.3 \%)$ and $4(7.6 \%)$, respectively.

The prevalence and distribution of 254 IR defects was calculated. Among them Grade I, II and III IR defects comprised 139 defects (54.7\%), 94 defects (37\%), 21 defects (8.3\%), respectively. It means that Grade I > II > III IR defect.

\subsection{Part B: arch}

The prevalence and distribution of absolute defects was evaluated in different arches. In the maxilla out of 529 teeth examined, 528 absolute defects were present, while in mandible out of 512 teeth examined 482 absolute defects were present. Fig. 1 depicts the prevalence and distribution of absolute defects in the arches.

The differences in the results for absolute bone defects in different arches were found to be statistically significant. The prevalence and distribution of IB defects by arch i.e. for CR in maxilla were 59 defects (34.1\%), while IT defects were 114 defects (65.9\%). In the mandible 88 defects (62.4\%) were CR, while IT defects formed 53 defects (37.6\%). Using Fisher's exact test, $\mathrm{p}$ value as $<0.001$, hence statistically highly significant results.

The prevalence and distribution of IT defects in maxilla were 23 
Prevalence and distribution of absolute defects (SB, IB \& OB) according to arch

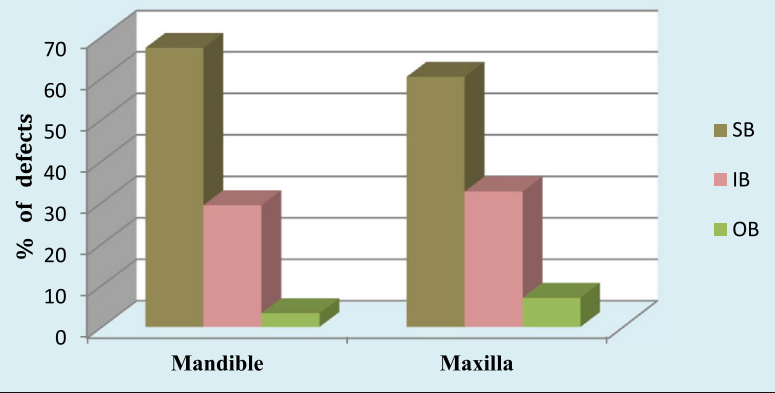

Fig. 1. Prevalence and distribution of absolute defects (Sb, Ib \& Ob) according to arch.

(20.2\%), 53 (46.5\%), 28 (24.6\%), $6(5.3 \%)$ and $4(3.5 \%)$, respectively and in mandible, it was 10 (18.8\%), 15 (28.3\%), 8 (15.1\%), 17 (32.1\%) and $3(5.7 \%)$, respectively (statistically highly significant results).

The prevalence and distribution of $\mathrm{OB}$ defects according to arch i.e. maxilla and mandible for BB, LD, RA, FN, DH, TR and RM defects were 10 (27\%), 17 (45.9\%), 2 (5.4\%), 0, 1 (2.7\%), 3 (8.1\%) and $4(10.8 \%)$, respectively and in mandible they were $4(25 \%), 5(31.3 \%), 1(6.3 \%)$, $0,3(18.8 \%), 3(18.8 \%)$ and 0 , respectively (statistically not significant).

The prevalence and distribution of IR defects i.e. Grade I, II and III IR defects in maxilla were found to be 87 (58\%), 60 (40\%) and $3(2 \%)$, respectively, while in mandible they were $52(50 \%), 34$ (32.7\%) and 18 (17.3\%), respectively ( $\mathrm{p}$ value $<0.001$, highly significant).

It was observed that the prevalence of IR defects was Grade I $>$ II > III both for maxilla and mandible. The differences were clinically as well as statistically highly significant. The prevalence of IR defects was found to be $57.5 \%$ and $72.7 \%$ among multirooted teeth for maxilla and mandible, respectively.

\subsection{Part C: segments}

Out of the total 1010 defects, the prevalence and distribution of absolute defects (SB, IB and OB defects) in 4 different segments were tabulated and graphically represented in Fig. 2. It was observed that the prevalence of defects was highest for maxillary posterior and lowest for the mandibular anterior.

The prevalence and distribution of absolute defects (SB, IB and OB defects) was tabulated for anterior and posterior segments (Table 1). They further depicted the prevalence and distribution of defects in maxilla and mandible.

SB defects were found to be $48.2 \%$ in anterior segments, while in



Fig. 2. Prevalence and distribution of absolute defects (Sb, Ib \& Ob) according to segments. posterior teeth it was $51.8 \%$. Eventhough, there was no remarkable difference in prevalence and distribution of SB defects and they were equally distributed among the 4 different segments.

The IB defects were found be $14 \%$ for the anterior segments only, while in the posterior segments $86 \%$ of IB defects were found. It was observed that maxillary posteriors had highest prevalence and distribution for IB defects followed by mandibular posterior and thereafter, maxillary anterior and mandibular anterior.

The CR were found to be $4 \%$ for the anterior segments, while in the posterior segments $96 \%$ of CR were found. It was observed that mandibular posteriors had highest occurrence of craters, they accounted for half of the total craters in the present study population.

The IT defects were found be $22.8 \%$ for the anterior segments, while in the posterior segments $77.2 \%$ of IT defects were found. It was observed that maxillary posteriors had highest occurrence of IT defects.

It was observed that maxillary posterior had highest occurrence of all the IT defects i.e. 1-, 2-, 3-, 4-wall and CB defect, followed by mandibular posterior and thereafter, maxillary anterior and mandibular anterior. Also, majority of 1-, 2-, 3-, 4-wall and CB defect were found in posterior segments than the anterior segments.

The OB defects were $11.4 \%$ in anterior segment, while they were $88.6 \%$ in posterior segments. It was observed that maxillary posteriors had highest prevalence and distribution of all the OB defects. Also, OB defects were more common in the posterior segment than anterior segment. Majority of BB, LD, RA, TR and RM were found in posterior segments while DH was present equally both for anterior and posterior segment.

The prevalence and distribution of various IB defects i.e. CR and IT, were observed for all the 4 different segments and has been depicted in Table 2 . The differences were clinically as well as statistically highly significant.

The prevalence and distribution of various OB defects was observed for all 4 different segments and has been depicted in Table 3.

\section{Discussion}

Epidemiology is not purely a descriptive science, but it is also a constructive science. There is a need to apply the epidemiological principles to periodontal bone defects and furnish data on prevalence and distribution of these defects.

In order to overcome the various limitations of all the procedures surgical inspection was done in the present study, which is considered to be the gold standard for the evaluation of periodontal bone defects. ${ }^{4}$

\subsection{Part A: study population}

It was observed that the occurrence of $\mathrm{SB}>\mathrm{IB}>\mathrm{OB}$ defects. Study by Persson RE et al. $1998^{5}$ was in agreement with the present study and they found horizontal bone defects to be $91 \%$ and vertical bone defects were $9 \%$ on radiographic examination. ${ }^{5}$ The pattern of distribution that horizontal bone defects were dominant than vertical bone defects, was similar to our finding but the differences in percentages was large.

Interdental crater was the commonest infrabony defect confirms the previous studies by Saari J et al. $1968^{6}$; Manson et al. $1974^{7},{ }^{8}$; Tal; $1984^{9}$; Vrotsos JA et al. $1999^{10}$ and Suefang Kung Wu et al. 2001. ${ }^{11}$

In the present study, CR accounted almost half of the total IB defects which was in agreement with the findings of Vrotsos JA et al. 1999. ${ }^{10}$

The prevalence and distribution of IT defects i.e. 1-, 2-, 3-, 4-wall and CB defect was as follows: $19.7 \%, 40.7 \%, 21.6 \%, 13.8 \%$ and $4.2 \%$, respectively. In the present study, among the IT defects the prevalence of defects was 2-wall $>3$-wall $>1$-wall $>4$-wall $>$ CB defect. While, in the study by Tal H; $1984^{9}$ it was found that among intrabony defects the prevalence of defects was 2-wall $>1$-wall $>3$-wall defect. Hence, it could be appreciated that 2 -wall defect was the dominant defect for both the studies but there was difference in sequence of other IT defects 
Table 1

Prevalence and distribution of absolute defects ( $\mathrm{Sb}$, Ib \& Ob) according to arch.

\begin{tabular}{|c|c|c|c|c|c|c|c|c|c|c|}
\hline \multirow[t]{2}{*}{ DEFECTS } & \multicolumn{4}{|c|}{ ANTERIOR } & \multicolumn{4}{|c|}{ POSTERIOR } & \multirow[t]{2}{*}{ Total } & \multirow[t]{2}{*}{$\%$} \\
\hline & MAX. & $\%$ & MAND & $\%$ & MAX. & $\%$ & MAND. & $\%$ & & \\
\hline SB & 153 & 23.8 & 157 & 24.4 & 165 & 25.7 & 168 & 26.1 & 643 & 100 \\
\hline IB & 29 & 9.2 & 15 & 4.8 & 144 & 45.9 & 126 & 40.1 & 314 & 100 \\
\hline a)CR & 1 & 0.6 & 5 & 3.4 & 58 & 39.5 & 83 & 56.5 & 147 & 100 \\
\hline b)IT & 28 & 16.8 & 10 & 6 & 86 & 51.5 & 43 & 25.7 & 167 & 100 \\
\hline 1 wall & 8 & 24.2 & 4 & 12.1 & 15 & 45.5 & 6 & 18.2 & 33 & 100 \\
\hline 2 wall & 11 & 16.2 & 1 & 1 & 42 & 61.8 & 14 & 20.6 & 68 & 100 \\
\hline 3 wall & 5 & 13.9 & 2 & 5.6 & 23 & 63.9 & 6 & 16.7 & 36 & 100 \\
\hline 4 wall & 3 & 13 & 2 & 8.7 & 3 & 13 & 15 & 65.2 & 23 & 100 \\
\hline $\mathrm{CB}$ & 1 & 14.3 & 1 & 14.3 & 3 & 42.9 & 2 & 28.6 & 7 & 100 \\
\hline OB & 4 & 7.6 & 2 & 3.8 & 33 & 62.2 & 14 & 26.4 & 53 & 100 \\
\hline $\mathrm{BB}$ & 0 & 0 & 0 & 0 & 10 & 71.4 & 4 & 28.6 & 14 & 100 \\
\hline LD & 2 & 9.1 & 0 & 0 & 15 & 68.2 & 5 & 22.7 & 22 & 100 \\
\hline RA & 0 & 0 & 0 & 0 & 2 & 66.7 & 1 & 33.3 & 3 & 100 \\
\hline DH & 0 & 0 & 2 & 50 & 1 & 25 & 1 & 25 & 4 & 100 \\
\hline TR & 1 & 16.7 & 0 & 0 & 2 & 33.3 & 3 & 50 & 6 & 100 \\
\hline $\mathrm{RM}$ & 1 & 25 & 0 & 0 & 3 & 75 & 0 & 0 & 4 & 100 \\
\hline Total & 186 & 18.4 & 174 & 17.2 & 342 & 33.9 & 308 & 30.5 & 1010 & 100 \\
\hline
\end{tabular}

which might be due to different study design and study population.

Among the $\mathrm{OB}$ it was inferred that $\mathrm{LD}>\mathrm{BB}>\mathrm{TR}>\mathrm{DH}=\mathrm{RM}>\mathrm{RA}$. These findings of present study, were similar with the finding of Manson et al. $1974^{7}$ where alveolar marginal defects (BB and LD) were found to be more common than perforations (FN and $\mathrm{DH}$ ).

In the present study, 254 IR defects were detected, and among them Grade I, II and III IR defect comprised 54.7\%, 37\% and 8.3\%, respectively. It can be inferred that Grade I > II > III IR defect. Grade I IR defect comprised half of the IR defects of the study population.

The explanation for such finding could be due to differences in the patient selection as in the present study, average age of patient selected was 36.8 years. Hence, the study sample was more accentuated towards younger age groups and therefore, less severity of disease can be seen. This finding was also in agreement with most epidemiological surveys which have shown a positive correlation between aging and severity of periodontal disease.

\subsection{Part B: $\operatorname{arch}$}

It was important to evaluate the distribution of defects between arches i.e. for maxilla and mandible as these differences provide clues for the pathogenesis of periodontal disease and add to the literature the variations in occurrences of defects among maxilla and mandible.

The absolute defects in maxilla were found to be more than the mandible. The possible explanation for the finding can be that, the major difference in the structure of maxilla, which is predominantly cancellous and with thin cortical plates, which enhances the formation of periodontal bone defect. ${ }^{8}$

The SB, IB and OB defects in maxilla were $60.2 \%, 32.8 \%$ and $7 \%$ defects, respectively, while for mandible they were $67 \%, 29.3 \%$ and $3.3 \%$ defects, respectively. Also, these differences in their occurrences were found to be clinically as well as statistically significant.

There was great diversity of defects which was seen in maxilla than mandible. In the present study and other supporting studies like by Manson JD 1974, ${ }^{7}$ the maxilla does appear to demonstrate a much greater diversity of bone deformities than the mandible, where interdental crater and 4-wall defect were the only relatively common defects. The possible explanation can be that the major difference between the maxilla \& the mandible is a structural one. Sicher (1949) pointed out that the lower alveolar process in most area is far stronger than that of the upper jaw, "only in the incisor \& cuspid area are the outer \& inner plates of the (mandibular) alveolar process thin". ${ }^{8}$ While the study by Vrotsos JA et al. $1999^{10}$ was not in agreement with our study as they found more defects per tooth in mandible (22.4\%) than in maxilla (15.4\%).

The prevalence and distribution of IB defects by arch i.e. for CR in maxilla were $34.1 \%$, while IT defects were $65.9 \%$. In the mandible $62.4 \%$ were CR, while IT defects formed $37.6 \%$. It was observed that the occurrences of IT defect in maxilla were double than CR and vice versa was true for mandible. The differences were clinically as well as statistically highly significant. These findings were similar with the Manson JD (1974) ${ }^{7}$ in which IT defects including hemisepta were found almost exclusively in the maxilla and mandible had craters as the predominant defect.

It was observed that 2-wall and 3-wall defects were dominant in

Table 2

Prevalence and distribution of Ib defects according to segments.

\begin{tabular}{|c|c|c|c|c|c|c|c|c|c|c|}
\hline \multirow[t]{3}{*}{ Defect } & \multicolumn{4}{|c|}{ MAXILLA } & \multicolumn{4}{|c|}{ MANDIBLE } & \multirow[t]{3}{*}{ TOTAL } & \multirow[t]{3}{*}{$\%$} \\
\hline & \multicolumn{2}{|c|}{ ANTERIOR } & \multicolumn{2}{|c|}{ POSTERIOR } & \multicolumn{2}{|c|}{ ANTERIOR } & \multicolumn{2}{|c|}{ POSTERIOR } & & \\
\hline & $\mathrm{T}$ & $\%$ & $\mathrm{~T}$ & $\%$ & $\mathrm{~T}$ & $\%$ & $\mathrm{~T}$ & $\%$ & & \\
\hline Crater & 1 & 3.5 & 58 & 40.3 & 5 & 33.3 & 83 & 65.9 & 147 & 46.8 \\
\hline 1 wall & 8 & 27.6 & 15 & 10.2 & 4 & 26.7 & 6 & 4.8 & 33 & 10.5 \\
\hline 2 wall & 11 & 37.9 & 42 & 29.2 & 1 & 6.7 & 14 & 11.1 & 68 & 21.7 \\
\hline 3 wall & 5 & 17.2 & 23 & 16 & 2 & 13.3 & 6 & 4.8 & 36 & 11.5 \\
\hline 4 wall & 3 & 10.3 & 3 & 2.1 & 2 & 13.3 & 15 & 11.9 & 23 & 7.3 \\
\hline $\mathrm{CB}$ & 1 & 3.5 & 3 & 2.1 & 1 & 6.7 & 2 & 1.6 & 7 & 2.2 \\
\hline Total DF & 29 & 100 & 144 & 100 & 15 & 100 & 126 & 100 & 314 & 100 \\
\hline
\end{tabular}

$\chi^{2}=74.63 ; \mathrm{d}(\mathrm{f})=15$.

$\mathrm{p}$ value $=<0.001$. Statistically highly significant. 
Table 3

Prevalence and distribution of $\mathrm{Ob}$ defects according to segments.

\begin{tabular}{|c|c|c|c|c|c|c|c|c|c|c|}
\hline \multirow{3}{*}{ Defect } & \multicolumn{4}{|c|}{ MAXILLA } & \multicolumn{4}{|c|}{ MANDIBLE } & \multirow[t]{3}{*}{ TOTAL } & \multirow[t]{3}{*}{$\%$} \\
\hline & \multicolumn{2}{|c|}{ ANTERIOR } & \multicolumn{2}{|c|}{ POSTERIOR } & \multicolumn{2}{|c|}{ ANTERIOR } & \multicolumn{2}{|c|}{ POSTERIOR } & & \\
\hline & $\mathrm{T}$ & $\%$ & $\mathrm{~T}$ & $\%$ & $\mathrm{~T}$ & $\%$ & $\mathrm{~T}$ & $\%$ & & \\
\hline BB & 0 & 0 & 10 & 30.3 & 0 & 0 & 4 & 28.6 & 14 & 26.4 \\
\hline LD & 2 & 50 & 15 & 45.5 & 0 & 0 & 5 & 35.7 & 22 & 41.5 \\
\hline RA & 0 & 0 & 2 & 6.1 & 0 & 0 & 1 & 7.1 & 3 & 5.7 \\
\hline FN & 0 & 0 & 0 & 0 & 0 & 0 & 0 & 0 & 0 & 0 \\
\hline DH & 0 & 0 & 1 & 3 & 2 & 100 & 1 & 7.1 & 4 & 7.5 \\
\hline TR & 1 & 25 & 2 & 6.1 & 0 & 0 & 3 & 21.3 & 6 & 11.3 \\
\hline $\mathrm{RM}$ & 1 & 25 & 3 & 9.1 & 0 & 0 & 0 & 0 & 4 & 7.5 \\
\hline Total & 4 & 100 & 33 & 100 & 2 & 100 & 14 & 100 & 53 & 100 \\
\hline
\end{tabular}

$\chi^{2}=25.87 ; \mathrm{d}(\mathrm{f})=5$.

$\mathrm{p}$ value $=<0.001$. Statistically highly significant.

maxilla, while in mandible 4-wall and 2-wall defects were dominant. The prevalence of 1-wall defect was same for both maxilla and mandible. The differences were clinically as well as statistically highly significant.

Almost two-third of OB defects were present in maxilla, while almost one-third of these defects were present in mandible. It was also seen that in maxilla the $\mathrm{BB}, \mathrm{LD}$ and $\mathrm{RM}$ were found to be more, while in mandible BB, LD, DH and TR were more common. The differences were clinically significant but statistically not significant. Studies by Rupprecht RD et al. $2001^{12}$ was in agreement as they also found DH to be more common in mandible than maxilla.

The prevalence of IR defects was Grade I > II > III both for maxilla and mandible. Also, the occurrences of Grade I and Grade II IR defects were more in maxilla, while the prevalence of Grade III IR defects were more in mandible. In the mandible the occurrence of Grade III IR defects was increased significantly from $2 \%$ in maxilla to $17.3 \%$ in mandible. The differences were clinically as well as statistically highly significant. This finding was according to finding of Manson; 1976, where 0.7 IR defects per segment were found in mandible and 0.54 IR defects were found for maxilla. This was contrasting with Svardstom et al. $1996^{13}$ and Papapanou; $1988^{14}$ which might be due to the differences in occurrences might be due to difference in study design and study population.

\subsection{Part C: segments}

The prevalence and distribution of absolute defects (SB, IB and OB defects) in 4 different segments were explored and was highest for the maxillary posterior i.e. $107 \%$, followed by mandibular posterior $98.7 \%$, thereafter in the anterior segments the maxillary anterior had the prevalence of $88.7 \%$, while the lowest prevalence was for the mandibular anterior i.e. $87 \%$.

\subsubsection{Suprabony defect}

Considering the total 643 SB defects to be $100 \%$, defects in maxillary anterior comprised of $23.8 \%$ in 210 teeth, in mandibular anterior defects comprised of $24.4 \%$ in 200 teeth, in maxillary posterior defects comprised of $25.7 \%$ in 319 teeth and mandibular posterior comprised of $26.1 \%$ of SB defects in 312 teeth. SB defects were found to be $48.2 \%$ in anterior segments, while in posterior teeth they were $51.8 \%$.

\subsubsection{Infrabony defect}

Considering the total 314 IB defects to be $100 \%$, maxillary anterior comprised of $9.2 \%$ of defects, mandibular anterior comprised of $4.8 \%$ of defects, maxillary posterior comprised of $45.9 \%$ of defects and mandibular posterior comprised of $40.1 \%$ of IB defects. It was observed that maxillary posterior had highest prevalence and distribution for IB defects. This showed that majority of IB defects to be in posterior segments than the anterior segments.

A significant difference in the prevalence and distribution of IB defect was found between anterior and posterior segments. This can be explained on the basis of thickness of alveolar process. The thick alveolar process in the posterior area provides an environment for the development of vertical defects. Waerhaug $1979\left({ }^{15}\right)$ suggested that the radius of action was approx. $1.5-2 \mathrm{~mm}$. Therefore, it is reasonable to speculate that bony plates thinner than $1.5-2 \mathrm{~mm}$ would be destroyed by microbial plaque attached before an angular defect would develop, while thicker bony plates would allow the development of vertical defects.

It was observed that mandibular posteriors had highest occurrence of craters. In the present study, it can be stated that almost half of IT defects were present in maxillary posterior, while they were least commonly present in mandibular anterior region. These findings of the present study, were similar with the findings with Manson JD et al. $1974^{7}$ where IT defects were found almost exclusively in the maxilla and they were least prevalent for mandibular anterior area.

It was observed that maxillary posterior had highest occurrence of all the IT and CB defects, followed by mandibular posterior and thereafter, maxillary anterior and mandibular anterior. Also, majority of IB defects were found in posterior segments than the anterior segments.

The arch and segment-wise prevalence and distribution of IB defects was determined in order to find out the prevalence of various IB defects in each segment. In the maxillary anterior frequency of defects in descending order was 2 -wall $>1$-wall $>3$-wall $>4$ wall $>$ crater $=\mathrm{CB}$. In the maxillary posterior frequency of defects in descending order was crater $>2$-wall $>3$-wall $>1$-wall $>4$ wall $=\mathrm{CB}$. In the mandibular anterior frequency of defects in descending order was crater $>1$-wall $>3$-wall $=4$-wall $>2$-wall $=\mathrm{CB}$. In the mandibular posterior frequency of defects in descending order was crater $>4$-wall $>2$-wall $>1$-wall $=3$-wall $>\mathrm{CB}$. The differences were clinically as well as statistically highly significant.

The finding by Vrotsos JA et al. $1999^{10}$ were that in the maxillary anterior frequency of defects in descending order was crater $>3$ wall $>2$-wall $>1$-wall $>4$-wall $>$ hemiseptum; in the maxillary posterior frequency of defects in descending order was crater $>4$ wall $>2$-wall $>1$-wall $>3$-wall $>$ hemiseptum; in the mandibular anterior frequency of defects in descending order was crater $>3$ wall $>2$-wall $=1$-wall $>$ hemiseptum $>4$-wall; in the mandibular posterior frequency of defects in descending order was crater $>3$ wall $>4$-wall $>2$-wall $=1$-wall $>$ hemiseptum. Crater was the dominant defect for all the segments for his study but in the present study it was the dominant defect for all the segments except for maxillary anterior where 2-wall defect was the dominant defect.

The interdental crater was found to represent almost $1 / 2$ of all the recorded defects, almost $2 / 3$ of mandibular posterior defects and 
almost $1 / 2$ of the defects in mandible; craters occurred frequently in both arches and most frequently in mandibular posterior segments. This finding accords with the finding of Vrotsos JA et al. $1999^{10}$

\subsubsection{Other bone defects}

Considering the total 53 OB defects to be $100 \%$, maxillary anterior had $7.6 \%$, mandibular anterior had $3.8 \%$, maxillary posterior had $62.2 \%$, while mandibular posterior had $26.4 \%$. The OB defects were $11.4 \%$ in anterior segment, while they were $88.6 \%$ in posterior segments.

It was observed that maxillary posteriors had highest occurrence of all the OB defects i.e. BB, LD, RA, TR and RM (except for DH which was most dominant defect in mandibular anteriors) Also, majority of BB, LD, RA, TR and RM were found in posterior segments than the anterior segments. Only DH was present equally both for anterior and posterior segment.

The prevalence and distribution of various $\mathrm{OB}$ defects was observed for 4 different segments (Table 3). It was also observed that for maxillary anterior frequency of defects in descending order was LD > TR $>$ RM. For the maxillary posterior frequency of defects in descending order $\mathrm{LD}>\mathrm{BB}>\mathrm{RM}>\mathrm{TR}=\mathrm{RA}>\mathrm{DH}$. For the mandibular anterior, frequency of defects in descending order OB defect explored was exclusively $\mathrm{DH}$ and in mandibular posterior, there were $\mathrm{LD}>\mathrm{BB}>\mathrm{TR}>\mathrm{RA}=\mathrm{DH}$. The differences were clinically as well as statistically highly significant.

Among the OB defects, BB and LD were predominantly present in maxillary posterior segment. These findings were similar with Manson JD et al. (1974) ${ }^{7}$; O'Connor et al. (1968), Horning GM et al. (2000) ${ }^{16}$ where thickened margins were more frequently found in maxilla than in the mandible and that too in the maxillary posterior segment. Possible explanation for formation of bulbous bone contour and ledge predominantly in maxillary posteriors can be:

1. Related to the high rate of remodeling possible in the maxilla

2. Greater reactivity of thin cortical plates of maxillary bone.

RA was found to be more common in maxillary posterior segment, while they were absent in anterior region. TR was dominant in mandibular posterior segment, while RM was commoner in maxillary posterior segment.

DH was found to be more common in mandibular anterior segment in present study this was similar finding by Rupprecht RD et al. $2001 .^{12}$

\section{Conclusion}

For the today's periodontists and patients, regeneration of the periodontium lost by periodontitis is an ultimate goal. The high prevalence as well as great variation and complexity of bone defects support the view that treatment procedures with predictable success rates are of great significance in periodontal therapy.

\section{Declaration of competing interest}

No conflict of interest.

\section{References}

1. Burt BA. Periodontitis and aging: reviewing recent evidence. $J$ Am Dent Assoc. 1994;125:273-279.

2. Ammons Jr WF, Harrinton GW. Furcation: involvement and treatment. In: Newman MG, Takei HH, Klokkevold PR, eds. Carranza FA." Carranza's Clinical Periodontology $10^{\text {th }}$ ed. Missouri: Saunder's; 2006:991-1004.

3. Lorato DC. Furcation involvements: incidence and distribution. J Periodontol. 1970;41:499-501.

4. Eickholz P, Riess T, Lenhard M, Hassfeld S, Staehle HJ. Digital radiography of interproximal bone loss; validity of different filters. J Clin Periodontol. 1999;26:294-300.

5. Persson RE, Hollender LG, Laurell L, Persson GR. Horizontal alveolar bone loss and vertical bone defects in an adult patient population. J Periodontol. 1998;69:348-356.

6. Saari JT, Hurt WC, Bigg NL. Periodontal bony defects on the dry skull. J Periodontol. 1968;39:278-283.

7. Manson JD, Nickolson K. The distribution of bone defects in chronic periodontitis. $J$ Periodontol. 1974;45:88-92

8. Manson JD. Bone morphology and bone loss in periodontal disease. J Clin Periodontol. 1976;3:14-22.

9. Tal H. The prevalence and distribution of intrabony defects in dry mandibles. $J$ Periodontol. 1984;55:149-154

10. Vrotsos JA, Parashis AO, Theofanatos GD, Smulow JB. Prevalence and distribution of bone defects in moderate and advanced adult periodontitis. J Clin Periodontol. 1999;26:44-48.

11. Wu Suefang Kung, Yeh Hwey-Chin, Chan Chiu-Po. The prevalence and distribution of bone defects in patients with moderate to advanced periodontitis. Chang Gung Med J. 2001;24:423-430.

12. Rupprecht RD, Horning GM, Nicoll BK, Cohen ME. Prevalence of dehiscences and fenestrations in modern American skulls. J Periodontol. 2001;72:722-729.

13. Svardstrom G, Wennstrom JL. Prevalence of furcation involvements in patients referred for periodontal treatment. J Clin Periodontol. 1996;23:1093-1099.

14. Papapanou PN, Wennstrom JL, Grondahl K. Periodontal status in relation to age and tooth type. A cross sectional radiographic study. J Clin Periodontol. 1988;15:469-478.

15. Waerhaug J. The angular bone defects and its relationship to trauma from occlusion and downgrowth of subgingival plaque. J Clin Periodontol. 1979;6:61-82.

16. Horning GM, Cohen ME, Neils TA. Buccal alveolar exostoses: prevalence, characteristics and evidence for buttressing bone formation. $J$ Periodontol. 2000;71:1032-1042. 